\title{
Lean Python
}

\section{Learn Just Enough Python to Build Useful Tools}

\section{Paul Gerrard}




\title{
Lean Python: Learn Just Enough Python to Build Useful Tools
}

Paul Gerrard

Maidenhead, Berkshire, United Kingdom

ISBN-13 (pbk): 978-1-4842-2384-0

ISBN-13 (electronic): 978-1-4842-2385-7

DOI 10.1007/978-1-4842-2385-7

Library of Congress Control Number: 2016958723

\section{Copyright (C) 2016 by Paul Gerrard}

This work is subject to copyright. All rights are reserved by the Publisher, whether the whole or part of the material is concerned, specifically the rights of translation, reprinting, reuse of illustrations, recitation, broadcasting, reproduction on microfilms or in any other physical way, and transmission or information storage and retrieval, electronic adaptation, computer software, or by similar or dissimilar methodology now known or hereafter developed.

Trademarked names, logos, and images may appear in this book. Rather than use a trademark symbol with every occurrence of a trademarked name, logo, or image we use the names, logos, and images only in an editorial fashion and to the benefit of the trademark owner, with no intention of infringement of the trademark.

The use in this publication of trade names, trademarks, service marks, and similar terms, even if they are not identified as such, is not to be taken as an expression of opinion as to whether or not they are subject to proprietary rights.

While the advice and information in this book are believed to be true and accurate at the date of publication, neither the authors nor the editors nor the publisher can accept any legal responsibility for any errors or omissions that may be made. The publisher makes no warranty, express or implied, with respect to the material contained herein.

\author{
Managing Director: Welmoed Spahr \\ Lead Editor: Steve Anglin \\ Technical Reviewer: Michael Thomas \\ Editorial Board: Steve Anglin, Pramila Balan, Laura Berendson, Aaron Black, Louise Corrigan, \\ Jonathan Gennick, Robert Hutchinson, Celestin Suresh John, Nikhil Karkal, James Markham, \\ Susan McDermott, Matthew Moodie, Natalie Pao, Gwenan Spearing \\ Coordinating Editor: Mark Powers \\ Copy Editor: Teresa F. Horton \\ Compositor: SPi Global \\ Indexer: SPi Global \\ Artist: SPi Global
}

Distributed to the book trade worldwide by Springer Science+Business Media New York, 233 Spring Street, 6th Floor, New York, NY 10013. Phone 1-800-SPRINGER, fax (201) 348-4505, e-mail orders-ny@springer-sbm.com, or visit www.springeronline.com. Apress Media, LLC is a California LLC and the sole member (owner) is Springer Science + Business Media Finance Inc (SSBM Finance Inc). SSBM Finance Inc is a Delaware corporation.

For information on translations, please e-mail rights@apress.com, or visit wWw.apress.com.

Apress and friends of ED books may be purchased in bulk for academic, corporate, or promotional use. eBook versions and licenses are also available for most titles. For more information, reference our Special Bulk Sales-eBook Licensing web page at ww. apress. com/bulk-sales.

Any source code or other supplementary materials referenced by the author in this text are available to readers at wWw. apress. com. For detailed information about how to locate your book's source code, go to WwW. apress. com/source-code/. Readers can also access source code at SpringerLink in the Supplementary Material section for each chapter.

Printed on acid-free paper 


\section{Contents at a Glance}

About the Author ............................................................................ xi

About the Technical Reviewer ……................................................. xiii

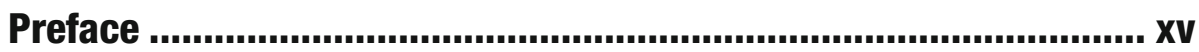

Acknowledgments ................................................................... xxiii

Chapter 1: Getting Started ............................................................ 1

Chapter 2: Python Objects ........................................................ 11

Chapter 3: Program Structure ..................................................... 25

Chapter 4: Input and Output ....................................................... 35

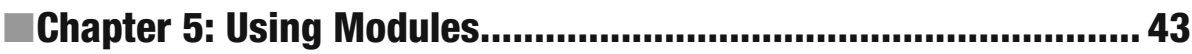

Chapter 6: Object Orientation ......................................................... 47

Chapter 7: Exception and Error Handling ........................................ 53

Chapter 8: Testing Your Code....................................................... 57

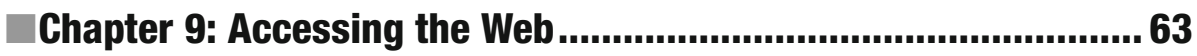

Chapter 10: Searching ............................................................67

Chapter 11: Databases ................................................................. 75

Chapter 12: What Next? ........................................................... 79

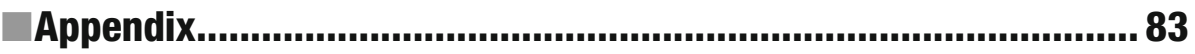

Index 


\section{Contents}

About the Author ........................................................................... xi

About the Technical Reviewer .................................................... xiii

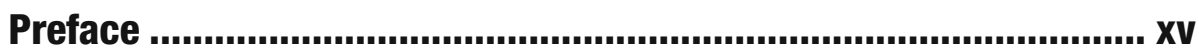

Acknowledgments ...................................................................... xxiii

Chapter 1: Getting Started......................................................... 1

The Python Interpreter …………………………………................ 1

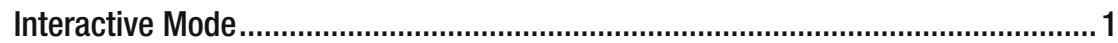

Command-Line Mode ...........................................................................................

Coding, Testing and Debugging Python Programs................................. 3

Comments, Code Blocks, and Indentation ............................................ 4

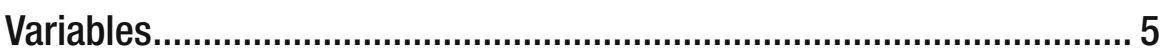

Common Assignment Operations ......................................................................

Other Assignment Operations .......................................................................... 6

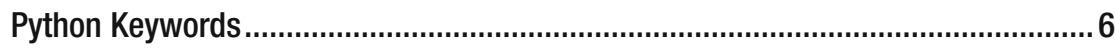

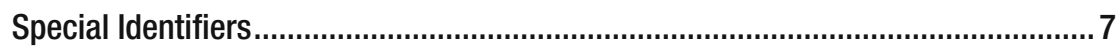

Python Modules................................................................................. 8

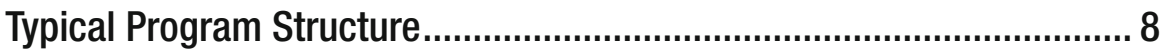

Chapter 2: Python Objects .......................................................... 11

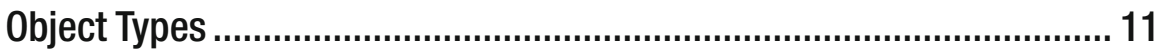

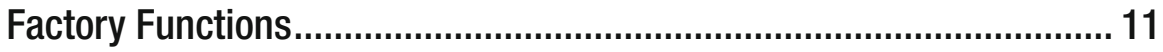

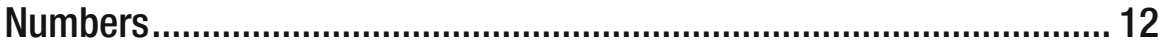




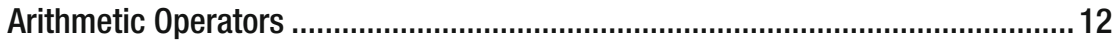

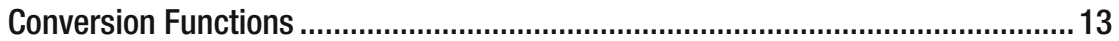

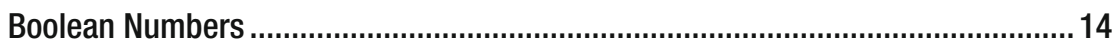

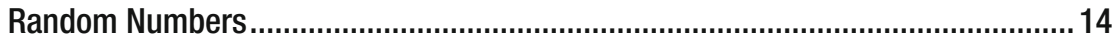

Sequences: Strings, Lists, and Tuples ................................................. 14

Sequence Storage and Access .........................................................................14

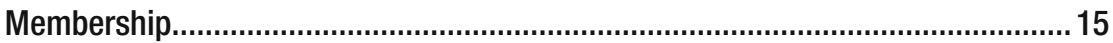

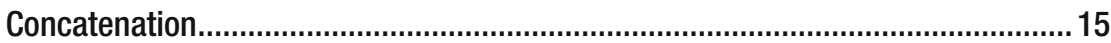

Sequence Elements and Slices ....................................................................16

Sequence Built-In Functions................................................................................ 16

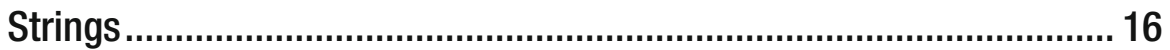

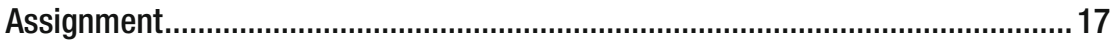

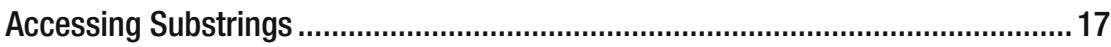

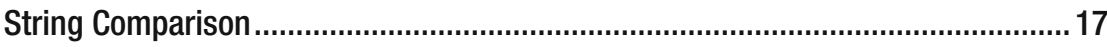

Membership (Searching) .............................................................................. 18

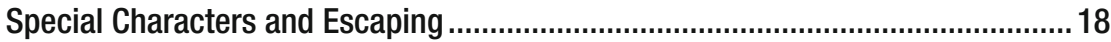

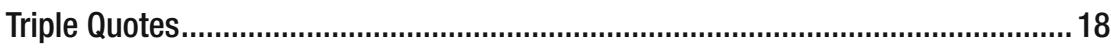

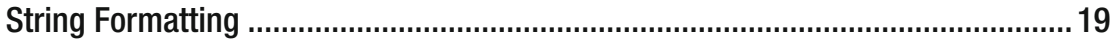

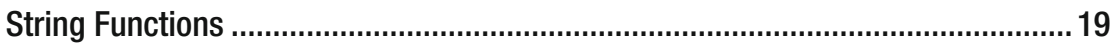

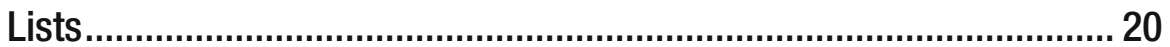

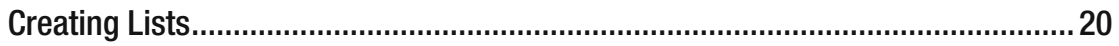

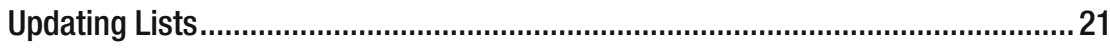

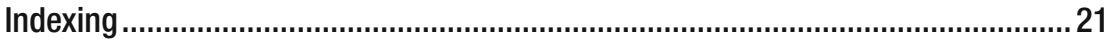

Sequence Operations and Functions .................................................................. 21

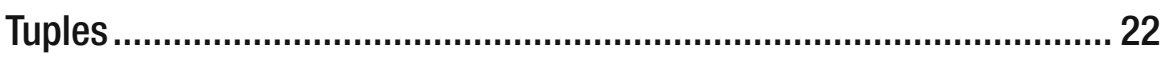

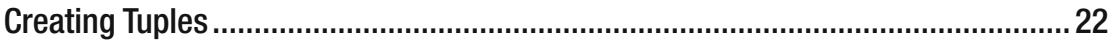

Sequence Operations and Functions .............................................................. 22

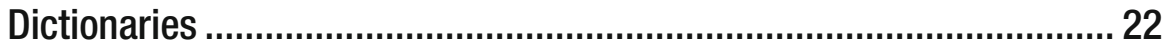

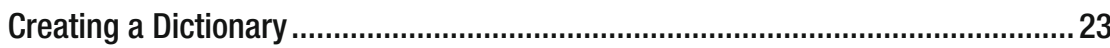

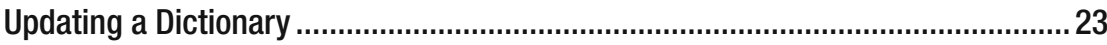

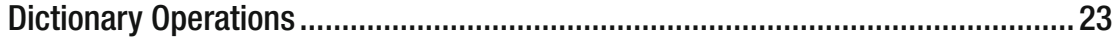


Chapter 3: Program Structure 25

Decision Making. 25

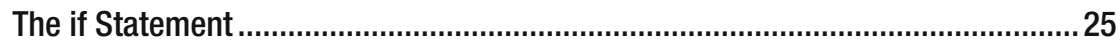

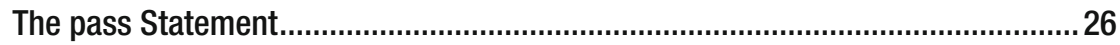

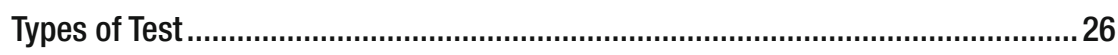

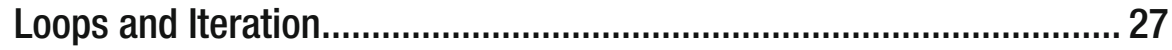

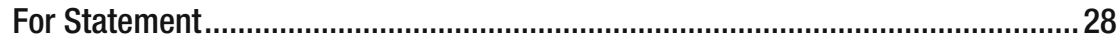

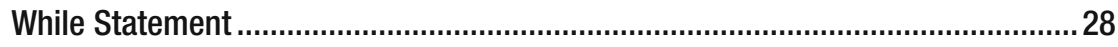

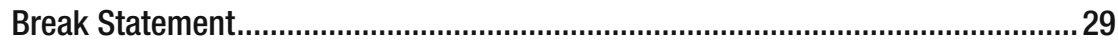

Continue Statement

List Comprehensions ................................................................................. 30

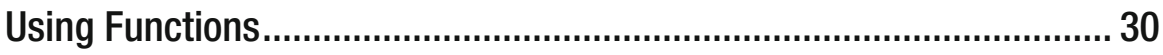

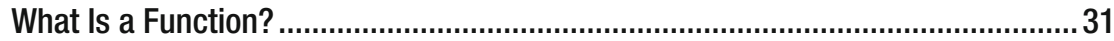

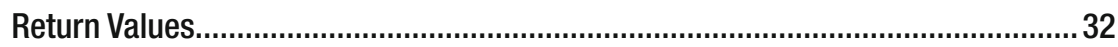

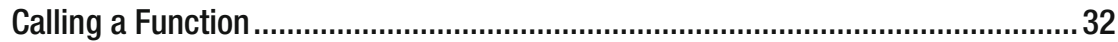

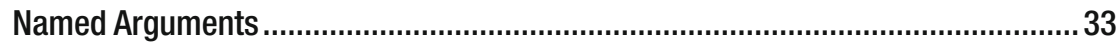

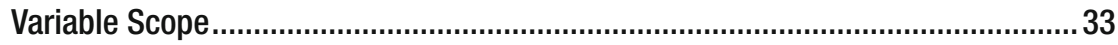

Chapter 4: Input and Output ....................................................... 35

Displaying Output.................................................................................... 35

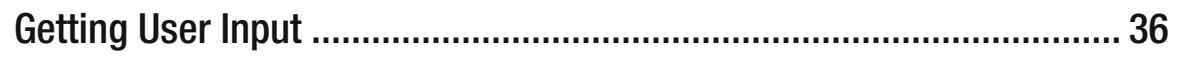

Writing and Reading Files .............................................................. 37

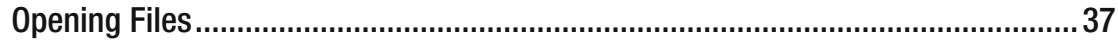

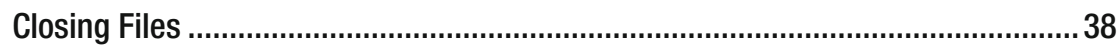

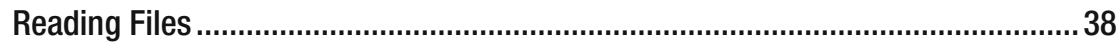

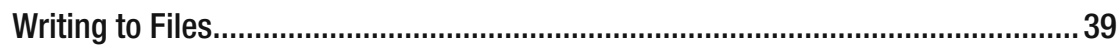

Accessing the File System.......................................................................... 40

Command-Line Arguments............................................................ 41 
Chapter 5: Using Modules........................................................... 43

Importing Code from a Module.............................................................. 43

Modules Come from the Python Path ................................................................. 43

Creating and Using Your Own Modules................................................................... 44

Chapter 6: Object Orientation .................................................... 47

What Is Object Orientation? ................................................................... 47

Creating Objects Using Classes............................................................. 48

Chapter 7: Exception and Error Handling ..................................... 53

Exceptions and Errors .................................................................... 53

Chapter 8: Testing Your Code................................................. 57

Modularizing Code and Testing It ......................................................5 57

Test-Driven Development ................................................................. 57

The unittest Framework .................................................................. 58

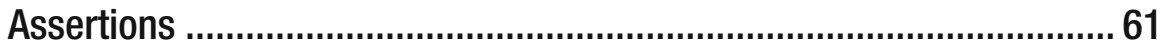

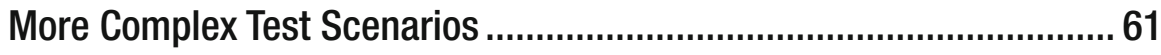

Chapter 9: Accessing the Web........................................................ 63

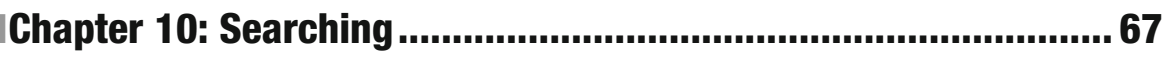

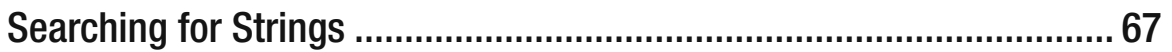

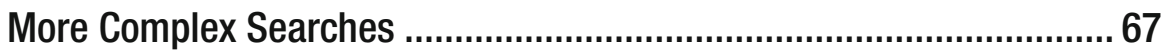

Introducing Regular Expressions.........................................................68

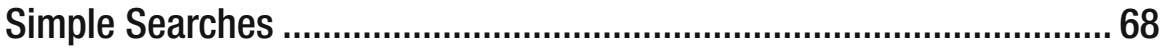

Using Special Characters ........................................................................ 68

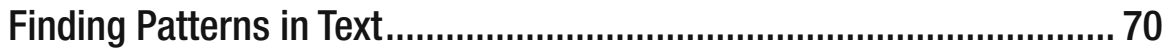

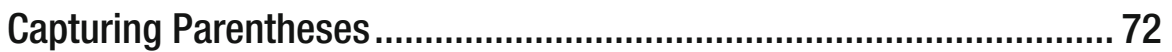

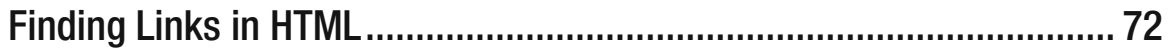


Chapter 11: Databases ............................................................... 75

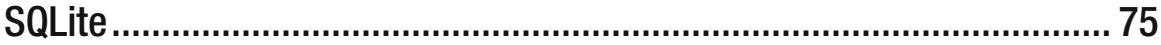

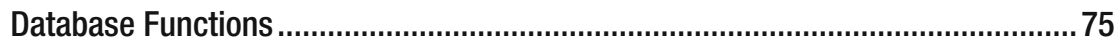

Connecting and Loading Data into SQLite ............................................. 76

Chapter 12: What Next? .................................................................... 79

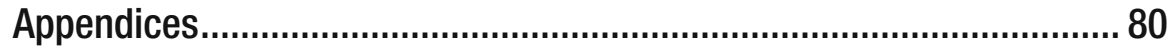

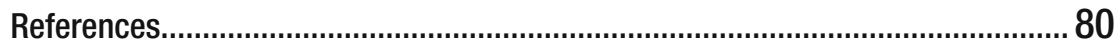

Python Built-In Exceptions Hierarchy ................................................................. 81

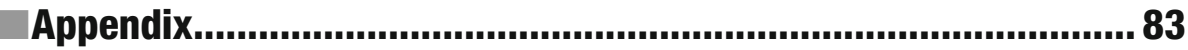

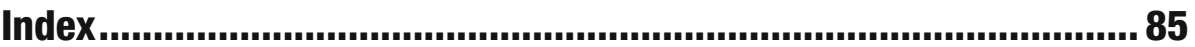




\section{About the Author}

Paul Gerrard is a consultant, teacher, author, webmaster, programmer, tester, conference speaker, rowing coach, and publisher. He has conducted consulting assignments in all aspects of software testing and quality assurance, specializing in test assurance. He has presented keynote talks and tutorials at testing conferences across Europe, the United States, Australia, and South Africa, and he has occasionally won awards for them.

Educated at the universities of Oxford and Imperial College London, he is a Principal of Gerrard Consulting Limited, the host of the UK Test Management Forum, and the Programme Chair for the 2014 EuroSTAR testing conference.

In 2010 he won the EuroSTAR Testing Excellence Award and in 2013 he won the inaugural TESTA Lifetime Achievement Award.

He has been programming since the mid-1970s and loves using the Python programming language. 


\section{About the Technical Reviewer}

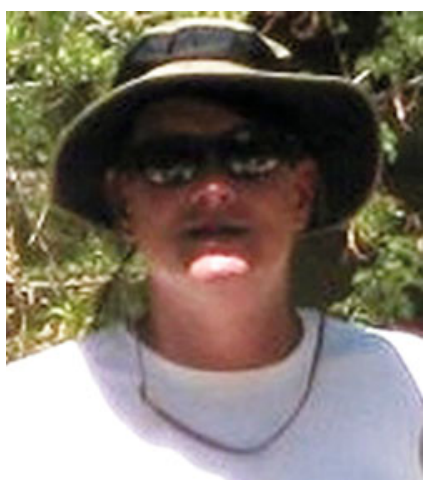

Michael Thomas has worked in software development for more than 20 years as an individual contributor, team lead, program manager, and vice president of engineering. Michael has more than 10 years of experience working with mobile devices. His current focus is in the medical sector, using mobile devices to accelerate information transfer between patients and health care providers. 


\section{Preface}

My first exposure to computer programming was at school nearly 40 years ago. My math teacher was a fan of computing and he established the first A-Level Computer Science course in the sixth form college. I didn't take the CS A-Level, as I was committed to Math, Physics, and Chemistry. But my math teacher invited all the scientists to do an informal class in programming, once a week, after hours. It sounded interesting, so I enrolled.

We were introduced to a programming language called CESIL, ${ }^{1}$ CESIL a cut-down version of an Assembler language ${ }^{2}$ with instructions that had more meaningful names like LOAD, STORE, ADD, and JUMP. We were given green cards on which the instructions and numbers were printed. Next to each instruction was a small oval shape. Beyond that, there was a shape for every letter and numeric value.

Filling in the shapes with a pencil indicated the instructions and data we wanted to use. To make the "job," work we topped and tailed our card deck with some standard instructions on more cards.

Our card decks were secured with rubber bands and sent off to Manchester University for processing. A week later, we usually (but not always) got our cards back together with a printout of the results. If we were lucky, our trivial programs generated some results. More often, our programs did not work, or did not even compile; that is, the computer did not understand our stumbling attempts to write meaningful program code.

I can't remember what programs I wrote in those days. Probably calculating squares of integers or factorials or if I was really ambitious, the sine of an angle using Taylor series. Looping (and more often, infinite looping) was a wonderful feature that had to be taken advantage of. Doing something that simply could not be done by humans was fascinating to me.

The challenge of thinking like the computer and of treating the mysterious machine in Manchester as an infallible wizard that must be obeyed-or at least communicated with in its own pedantic, arcane language-sticks in my mind. You could, with some practice, treat the wizard as your very own tireless slave. Those after-hours classes were great and I looked forward to them every week.

Programming was great fun, if you had a certain interest in control, procedure, and systematic thinking. Nearly 40 years later, I still enjoy battling with code. My programming language of choice nowadays is Python. ${ }^{3}$

${ }^{1}$ Computer Education in Schools Instruction Language (see http://en.wikipedia.org/wiki/ Cesil). If you are curious, you can download a fully working CESIL interpreter [18].

${ }^{2}$ Assembler is a very low-level language close to actual machine code.

${ }^{3}$ Throughout the book, I use the term Python as shorthand for "the Python programming language." 


\section{Introducing Python}

The Python programming language was created by Dutchman Guido van Rossum in the late 1980s [1]. Here is a concise summary of Python from Wikipedia [2]:

Python is a widely used, general-purpose, high-level programming language. Its design philosophy emphasizes code readability, and its syntax allows programmers to express concepts in fewer lines of code than would be possible in languages such as $C$. The language provides constructs intended to enable clear programs on both a small and large scale.

If you choose to learn Python as your first or your 15th programming language, you are making an excellent choice.

Of all the languages I have used (and I think it is about 15, over the years) Python is my favorite. I can't say exactly why, and I don't pretend to be an expert in these matters, but here are some of the things I like about Python:

- $\quad$ Programs are not cluttered up with braces $(\{\ldots\})$ and semicolons (;).

- $\quad$ Python implements structure using indentation (white space) rather than punctuation.

- $\quad$ The Python keywords are powerful, limited in number, and do what you expect them to do.

- If you can't work out a way to do something in your code, there is always a library somewhere that does it for you.

- You can get an awful lot done with a limited knowledge of the language'

It is this last feature that I like the most.

\section{Lean Python}

I freely admit that I don't know all the features of this wonderful language by heart. In that way, I am a less-than-perfect programmer and I beat myself up about it regularly. I have written about 40,000 lines of Python in the past five years, but I discovered recently that actually, I only need a distinct subset of the language to get things done. I do use all the core elements of the language, of course-lists, dictionaries, objects, and so on-but I don't (and can't) memorize all of the standard functions for each element. I haven't needed them.

I'm looking at a list of the functions and methods for sequences. There are 58 listed in my main Python source book [13]. I have only used 15 of them; I haven't found a need for the rest.

I call this subset Lean Python and it is all you need to know as a beginner and some way beyond. 
Note Lean Python is not "the best way to write code." I offer it as a way of learning the essential aspects of the language without cluttering up your mind with features you might never use.

Now, the code I have written with the Lean Python subset of language features means that on occasion, I have written less optimal code. For example, I discovered only recently that there is a reverse() function that provides a list in reverse order. Of course there is, and why wouldn't there be? Needless to say, I had overlooked this neat feature and have written code to access list elements in reverse order more than once.

These things happen to all programmers. In general, we don't consult the manual unless we have to, so it's a good idea, every now and then, to review the standard list of features for the language to see what might be useful in the future.

\section{Beyond Lean Python}

There are many excellent resources available that provide more comprehensive content than this little book. Web sites I would recommend as essential include these:

- $\quad$ python.org. This is the official site for the Python language, and often the best starting point.

- docs.python.org. This site provides the definitive documentation of the standard Python libraries.

There are several excellent sites that offer free, online tutorials. Of course, I also have my own; visit leanpy. com to access it.

Regarding books, there are three that sit on a shelf right above my desk at all times:

- $\quad$ Core Python Programming, by Wesley Chun

- $\quad$ The Python Standard Library by Example, by Doug Hellmann

- Python Cookbook, by Alex Martelli, Anna Ravenscroft, and David Ascher

There are many other excellent books, and you might find better ones, but these are the three that I use myself.

\section{Code Examples in the Book}

In this book, you will see quite a lot of example code. Early on you'll see some small code fragments with some narrative text. All code listings are presented in the Courier New font. The shaded text is the code, the unshaded text to the right provides some explanation. 
\#

\# some comments and code \# in here

\#

myName = 'Paul'

myAge $=21$ \# if only
Some explanation appears on the right-hand side.

Later on you'll see longer listings and whole programs. These appear in the book as shaded areas. Some listings have line numbers on the left for reference, but the line numbers are not part of the program code. For example:

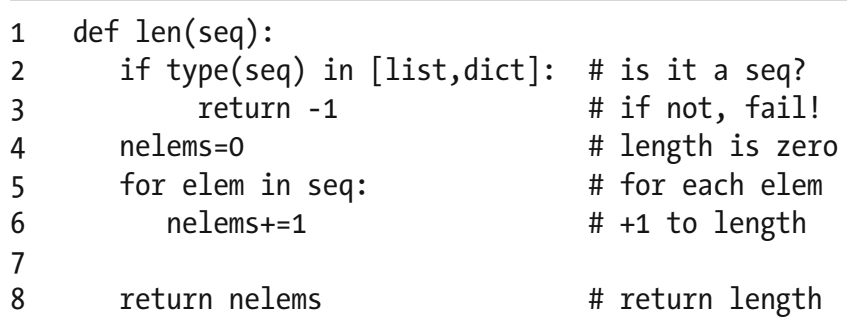

There are also some examples of interactions with the Python command-line shell. The shell gives you the $\gg>$ prompt. Here's an example:

〉> type(23)

$\langle$ type 'int'>

〉> type('some text')

〈type 'str' >

$\gg>c=[1,2$, 'some more text']

$\gg$ type $(\mathrm{c})$

〈type 'list'>

The lines are not numbered. The lines without the $\gg>$ prompt are the outputs printed by the shell.

Note Use the code fragments in the shaded sections to practice in the interactive interpreter or run the programs for yourself.

\section{Target Audience}

This book is aimed at three categories of readers:

- $\quad$ The experienced programmer: If you already know a programming language, this book gives you a shortcut to understanding the Python language and some of its design philosophy. 
- $\quad$ You work in IT and need a programming primer: You might be a tester who needs to have more informed technical discussions with programmers. Working through the examples will help you to appreciate the challenge of good programming.

- First-timer: You want a first book on programming that you can assimilate quickly to help you decide whether programming is for you.

If you require a full-fat, 1,000-page reference book for the Python language, this book is not for you. If you require a primer, appetizer, or basic reference, this book should satisfy your needs.

\section{What This Book Is}

This little book provides a sequential learning guide to a useful and usable subset of the Python programming language. Its scope and content are deliberately limited and based on my own experience of using Python to build interactive web sites (using the Web2py web development framework [3]) and many command-line utilities.

This book accompanies the one- and two-day programming courses that I created to help people grasp the basics of a programming language quickly. It isn't a full language reference book, but a reference for people in the course and for whom the Lean Python subset is enough (at least initially).

\section{What This Book Is Not}

This book is not intended to be a definitive guide to Python.

\section{Code Comprehension}

The initial motivation for writing this book was to help provide nontechnical (i.e., nonprogrammer) testers with an appreciation of programming so they could work more closely with the professional programmers on their teams. Critical to this is the skill I call code comprehension, which is your ability to read and understand program code.

Like spoken and written languages, it is usually easier to comprehend written language than write it from scratch. If the book helps you to appreciate and understand written program code, then the book will have succeeded in its first goal.

\section{Python Style Guidelines}

One of the most important attributes of code is that it is written to be read by people, not just computers. The Python community gives this goal a high priority. In your own company, you might already have programming or Python guidelines; the Python team have provided some that are widely used [4]. 
I have tried to follow the guidelines in the sample code and programs. However, in the pocket book format, there is less horizontal space, so sometimes I have had to squeeze code a little to fit it on the page. I tend to use mixed case, e.g., addTwoNumbers in my variable and function names. ${ }^{4}$

Some of my code comments, particularly in the early pages, are there to explain what, for example, an assignment does. You would not normally expect to see such "stating the obvious" comments in real code.

"Pythonistas" take the readability goal seriously, and so should you.

There is also a set of design principles you might consult. The Zen of Python sets them out [5]. I'm sure I could have written better examples; if you see an opportunity to improve readability or design, let me know.

\section{Structure}

The first seven chapters cover the core features of Python. The later chapters introduce some key libraries and how you can use them to write useful applications.

Chapter 1 introduces the interpreter, the basic syntax of the language, the normal layout, and the conventions of Python. Chapter 2 describes the core Python objects that you will use and need to understand. Chapter 3 sets out how programs are structured and controlled using decisions and loops. Chapter 4 tells you how to get data into and out of your programs with the command line, display, and disk files. Chapter 5 introduces modules that help you to manage your own code and access the thousands of existing libraries. Chapter 6 gives you a flavor for object orientation. Objects and classes are the key building blocks that programmers use. Chapter 7 presents methods for trapping errors and exceptions to allow your programs to be "under control" whatever happens.

Chapter 8 describes how you can use the unittest framework to test your code in a professional manner. Chapter 9 introduces libraries allowing you to create a web client and download pages from web sites. Chapter 10 presents regular expressions as the mechanism for more sophisticated searching and pattern-matching. Chapter 11 gives you techniques for creating and using the SQLite relational database for persistent storage. Chapter 12 asks "What Next?" and offers some suggestions for further development of your Python programming skills.

An Appendix contains references to web sites, books and tools, and the Python exception hierarchy. An index is included at the end of the book.

\section{Using Python Downloading Python}

All Python downloads can be found at https://www. python.org/downloads/.

You need to choose a Python version before you download. There are currently two versions:

- Version 2 is coming to the end of its life but is still widely used.

- Version 3 has been around for some time; people have been slow to convert but it is gaining a following.

\footnotetext{
${ }^{4}$ The guideline suggests lower_case_with_underscores.
} 
The example code in this book assumes you are using Version 3. If you use Python Version 2 you will notice a few differences. You can read a discussion of the two Python versions in [6].

\section{Sample Programs Download}

Downloadable sample programs can be found at http://leanpy.com/?page_id=37.

All the sample programs have been tested on Windows 8, Ubuntu Linux 13, and my trusty Raspberry Pi running Linux. If you use a Mac, you should not have problems.

\section{External Libraries}

A major benefit to using Python is the enormous range of free libraries that are available for use. The vast majority of these libraries can be found on the PyPI site [7]. When I last looked, there were 46,554 packaged libraries hosted there.

Depending on your operating system (Windows, Mac or Linux), there are several ways of performing installations of Python libraries. The one I find easiest to use is the PIP installer [19] which works nicely with the PyPI site.

\section{Editing Your Python Code}

I recommend using either a language-sensitive editor or the editor that comes with your Python installation.

- On Windows, use the IDLE Integrated Development Environment (IDE) or perhaps Notepad++.

- On Linux, there is a selection of editors-vi, vim, emacs, gedit, and so on; I use gedit.

- $\quad$ On OS X, TextMate works fine, but there are other options.

When you are more experienced, you might upgrade to using an IDE. There is a list of Python-compatible IDEs available at https://wiki.python.org/moin/ IntegratedDevelopmentEnvironments.

\section{Feedback, Please!}

I am very keen to receive your feedback and experience to enhance the format and content of the book. Give me feedback and I'll acknowledge you in the next edition.

Any errors or omissions are my fault entirely. Please let me know how I can improve this book. E-mail me at paul@gerrardconsulting. com with suggestions or errors.

Downloads, errata, further information, and a reading list can be found on the book's web site at leanpy.com. 


\section{Acknowledgments}

For their helpful feedback, guidance, and encouraging comments, I'd like to thank James Lyndsay, Corey Goldberg, Simon Knight, Neil Studd, Srinivas Kadiyala, Julian Harty, and Fahad Ahmed. 
"Everyone knows that debugging is twice as hard as writing a program in the first place. So if you're as clever as you can be when you write it, how will you ever debug it?"

-Brian W. Kernighan

"Talk is cheap. Show me the code"

- Linus Torvalds

"Programs must be written for people to read, and only incidentally for machines to execute"

-Abelson/Sussman

"First, solve the problem. Then, write the code"

-John Johnson

"Sometimes it pays to stay in bed on Monday, rather than spending the rest of the week debugging Monday's code"

-Dan Salomon

"This project is seriously ahead of schedule"

-Perplexed IT director

"The most disastrous thing that you can ever learn is your first programming language"

-Alan Kay 